\title{
Anti dyslipidemic effect of Ficus Carica Fruit (Fig) on Lipid Profile of Diet Induced Hyperlipidemic Rats
} \author{
ROBINA IQBAL 6 \\ ${ }^{1}$ Assistant Professor Pharmacology, Sharif Medical and Dental College, Lahore \\ ${ }^{2}$ Assistant Professor Pharmacology, Akhtar Saeed Medical and Dental College, Lahore \\ ${ }^{3}$ Assistant Professor Pharmacology, CMH Institute of Medical Sciences Bahawalpur \\ ${ }^{4}$ Assistant Professor Pharmacology, Post Graduate Medical Institute/Ameer-ud-Din Medical College, Lahore \\ ${ }^{5}$ Assistant Professor Pharmacology, Sharif Medical and Dental College, Lahore \\ ${ }^{6}$ Associate Professor Pharmacology, Post Graduate Medical Institute/Ameer-ud-Din Medical College, Lahore \\ Correspondence to: Fariha Ahmad Khan, Email: farihazeeshankhan@gmail.com, Cell: 03334457193
}

FOUZIA PERVEEN ${ }^{1}$, FARIHA AHMAD KHAN ${ }^{2}$, ZOOBIA IRUM ${ }^{3}$, AMIR HASSAN SIDDIQUI ${ }^{4}$, MAIRA BHATTI ${ }^{5}$,

\begin{abstract}
Back ground: Hyperlipidaemia, characterized by elevation of one or more lipid types in blood, is a major risk factor for cardiovascular diseases and stroke. Management of hyperlipidaemia is helpful to control the fatal outcome of cardiovascular diseases. This experimental study was done to observe the effects of pulp of Ficus carica fruit on lipid profile in induced hyperlipidemic rats in comparison to atorvastatin.

Methodology: The duration of study was 12 weeks. In this study forty male rats were taken and divided into four groups. One group was normal control which was given normal diet throughout study period of 12 weeks. The second group was positive control which was given high fat diet for 12 weeks. The remaining two groups were experimental groups which were given high fat diet for 12 weeks. Hyperlipidaemia was induced after giving high fat diet for first four weeks to the experimental groups. The pulp of Ficus carica fruit and atorvastatin was then added to diet of two experimental groups respectively in addition to high fat diet for the next 8 weeks. Body weight, fasting lipid profile, LDL/HDL ratio and AIP were measured at baseline, $4^{\text {th }}, 8^{\text {th }}$, and $12^{\text {th }}$ week of study.

Results: Pulp of Ficus carica fruit significantly improved fasting lipid profile, AIP and LDL/HDL ratio. HDL was also significantly increased. However there was no change in body weight.

Conclusion: Ficus carica fruit has marked antihyperlipidemic properties comparable to standard drug atorvastatin.
\end{abstract}

Key words: Hyperlipidaemia, ethanolic extract, pulp, Ficus carica

\section{INTRODUCTION}

Hyperlipidaemia is the main cause of cardiovascular disease. In 2005, statistical data showed 100,000 deaths due to cardiovascular disease in Pakistan ${ }^{1}$. Hyperlipidaemia is a major risk factor of stroke. Mortality due to stroke is more than 75,000 according to $\mathrm{WHO}$ in Pakistan².

Hyperlipidaemia is a disorder caused by increase of plasma lipids ${ }^{3}$. Hyperlipidaemia is classified as primary and secondary types. Primary hyperlipidaemia is due to environmental factors or genetic factors in the metabolism of lipids ${ }^{4}$. When hyperlipidaemia occurs due to metabolic disorders like chronic kidney disease, Cushing syndrome and diabetes mellitus, it is called as secondary hyperlipidemia ${ }^{5}$. Decrease in levels of serum lipids can be helpful to control hypertension, diabetes mellitus and coronary artery disease ${ }^{6}$

Treatment of hyperlipidaemia starts with life-style modifications including exercise and low-fat diet comprising more unsaturated fats. If not managed this way, pharmacotherapy includes use of statins, fibrates, niacin and bile acid sequestrants. Statins are first line and effective drugs but their adverse effects include myopathy and hepatotoxicity. They are contraindicated in pregnancy and hepatic dysfunction? ${ }^{7}$.

Natural herbs can be used for the management of hyperlipidaemia. Fig (Ficus carica Linn), belonging to

Received on 18-01-2021

Accepted on 15-06-2021
Moraecae family, belongs to one of largest genera of plants having medicinal value. Parts of Ficus carica have been studied due to their medicinal importance ${ }^{8}$. Analysis of of Ficus carica reveals that it contains alkaloids, xanthotoxols, glycosides, polyphenols, flavonoids and many other compounds. Along with other therapeutic uses including hypoglycemic, hepatoprotective, antispasmodic, antipyretic, anti HSV, antioxidant and antibacterial actions, hypolipidemic effect has also been shown by fruit and leaves of Ficus carica ${ }^{9}$. Ficus carica shows hypolipidemic effect on hepatic and serum cholesterol ${ }^{10}$.

The Ficus Carica fruit has hypolipidemic effects but the studies which were done previously used the different Ficus carica extracts. Whole fruit was not used in previous studies as a test agent. This study has been done to assess hypolipidemic effect of Ficus carica fruit on induced hyperlipidemic rats in comparison to atorvastatin, a standard drug used in dyslipidaemia. This will provide an easy-to-use treatment of hyperlipidaemia and better patient compliance due to lack of side effects.

\section{MATERIALS AND METHODS}

This 12-weeks experimental study was performed at Post Graduate Medical Institute, Lahore, Pakistan after approval from institutional ethical committee. The study was done from October 2016 to December 2016. Forty male Sprague Dawley rats weighing between $130-170$ grams were randomly divided into four groups. Animals were acclimatized for one week before start of study at a temperature of $\pm 25^{\circ} \mathrm{C}$ and 12 hours light dark circle with 
water and food given ad libitum. Rats were given proper care according to the already set citeria of 'Guide for use and care of laboratory animal'11.

Chemicals: Research grade chemicals manufactured by Sigma Aldrich, Germany were used in this research. Pharmaceutical grade atorvastatin powder was obtained from Schazoo Zaka pharmaceuticals, Lahore. Diagnostic kits were from Human, Germany. Coconut oil marketed by Parachute, India was used and dried fruit of Ficus carica was purchased from the local market.

Animal Groups: 40 male rabbits were taken and divided randomly into four groups, so one group has ten animals. One group was normal control, which was given normal diet throughout study duration of 12 weeks. The positive control group (HFD) was given high fat diet throughout study duration. The two experimental groups were also given high fat diet throughout study period. After 4 weeks, Ficus carica pulp was added to one experimental group (HFD+Ficus carica pulp) and atorvastatin was added to other experimental group (HFD+Atorvastatin) for next 8 weeks. The intervention from $5^{\text {th }}$ to $12^{\text {th }}$ week is shown in table 1.

Induction of Hyperlipidaemia: High fat diet containing 1 gm sodium cholate, $1.5 \mathrm{gm}$ cholesterol and $8 \mathrm{ml}$ of coconut oil per $100 \mathrm{gm}$ standard rat chow diet was given to positive control (HFD alone) and experimental groups throughout duration of study. It produced hyperlipidaemia within 4 weeks ${ }^{12}$.

Ficus carica pulp administration: After botanical verification from Government College University, Lahore, pellets of Ficus carica pulp were administered to HFD+FCP group as $1250 \mathrm{mg} / \mathrm{kg}$ single morning oral dose. This dose was calculated as follows to make it equivalent to extract dose used in similar studies ${ }^{13}$.

Dose of pulp given $=\frac{\text { total amount of fig }}{\text { yeild of extract }} \times$ dose of extract

Administration of Atorvastatin: Atorvastatin was administered to HFD+ATO group as $30 \mathrm{mg} / \mathrm{kg}$ daily single morning oral dose ${ }^{14}$.

Sampling and Parameters: Body weight was measured on $0,4,8$ and 12 weeks. Lipid profile was measured according to kit manufacturer's protocol on week $0,4,8$ and 12 from blood obtained through rats' cardiac puncture after fasting for 12 hours. Ratio of LDL/HDL, LDL and atherogenic index of plasma was calculated from these values.

Statistical Analysis: Statistical analysis was done using SPSS 22. Mean \pm SD were calculated; normality of data was checked with Shapiro-wilk and Kolmogorov test; ANOVA and post hoc Tukey test were applied to determine significant differences among all groups and within individual groups.

\section{RESULTS}

Body weight: Persistent and significant rise in body weight of rats in all the groups was observed with maximum increase being in HFD \pm ATO group which was followed by HFD alone group. The difference among the groups was, however, insignificant ( $p$ value 0.567 ) at the end of study.

Cholesterol: Highest in HFD alone group at end of the study, total level of cholesterol showed a persistent, highly significant, and comparable decrease in HFD+FCP and HFD+ATO groups in comparison to HFD alone group ( $p$ value $<0.001$ for both groups). It was numerically lower in HFD+FCP group than HFD+ATO group; the difference was, though, statistically insignificant.

Triglycerides: The highest serum triglyceride level was observed in HFD alone group. Rats in HFD+FCP and HFD+ATO groups showed a persistent and highly significant decline in level of serum TG compared to disease control at week 12 ( $p$ value $<0.001$ for both groups). These values were significantly lower than even normal control group ( $p$ value 0.004 for both groups).

HDL: HDL increase was observed at end of the study in all groups except normal control. The increase was maximum in HFD+FCP group which was significantly higher than HFD alone group ( $p$-value 0.016). HDL level in HFD+ATO group was higher than HFD alone group but lower than HFD+FCP group. The difference was, however, statistically insignificant in either case.

LDL: LDL level was highest in HFD alone group at the end of study. Atorvastatin and Ficus carica pulp both decreased it significantly than HFD alone group ( $p$ value $<0.001$ ). This decrease was the most marked in HFD+FCP group reaching to the level of statistical insignificance when compared to normal control group. HFD+ATO group exhibited significantly higher LDL than NC ( $p$ value $<0.001$ ).

Table 1: Animal grouping with diet given and interventions done throughout the study

\begin{tabular}{|l|l|l|}
\hline Group & Diet & $\begin{array}{l}\text { Intervention from 5-12 } \\
\text { weeks }\end{array}$ \\
\hline Normal control & $\begin{array}{l}\text { Normal rat } \\
\text { chow }\end{array}$ & Distilled water p.0. $2.5 \mathrm{ml} / \mathrm{kg}$ \\
\hline $\begin{array}{l}\text { High Fat Diet (HFD) } \\
\text { group }\end{array}$ & High fat diet & Distilled water p.o. $2.5 \mathrm{ml} / \mathrm{kg}$ \\
\hline $\begin{array}{l}\text { HFD + Ficus carica } \\
\text { pulp }\end{array}$ & High fat diet & $1250 \mathrm{mg} / \mathrm{kg} \mathrm{p.o.} \mathrm{fruit} \mathrm{pulp}$ \\
\hline HFD + atorvastatin & High fat diet & $30 \mathrm{mg} / \mathrm{kg}$ p.o. atorvastatin \\
\hline
\end{tabular}

Table 2: Results of Ficus carica pulp and atorvastatin treatment on body weight (grams) and lipid parameters (mg/dl) in rats. HFD - high fat diet, FCP - Ficus carica pulp; ATO - atorvastatin.

\begin{tabular}{|c|c|c|c|c|c|}
\hline & & Normal Control & HFD alone & HFD+FCP & HFD+ATO \\
\hline \multirow{3}{*}{ Body Wt. } & Week 0 & $137.38 \pm 7.63$ & $140.88 \pm 9.06$ & $139.75 \pm 9.54$ & $146.25 \pm 8.86$ \\
\hline & Week 4 & $212.00 \pm 32.16$ & $224.75 \pm 15.84$ & $229.63 \pm 16.75$ & $231.50 \pm 25.57$ \\
\hline & Week 12 & $292.75 \pm 15.75$ & $301.75 \pm 30.94$ & $295.50 \pm 35.75$ & $315.63 \pm 36.06$ \\
\hline \multirow{3}{*}{ Cholesterol } & Week 0 & $78.76 \pm 10.45$ & $81.75 \pm 5.1$ & $74.02 \pm 9.97$ & $82.12 \pm 8.93$ \\
\hline & Week 4 & $71.34 \pm 4.89$ & $190.01 \pm 50.54$ & $213.37 \pm 33.93$ & $207.25 \pm 55.89$ \\
\hline & Week 12 & $52.45 \pm 17.80$ & $207.95 \pm 39.33$ & $113.87 \pm 17.66^{* * *}$ & $136.62 \pm 18.16^{* \star *}$ \\
\hline \multirow{3}{*}{ TGs } & Week 0 & $85.44 \pm 4.08$ & $88.63 \pm 7.98$ & $94.25 \pm 5.17$ & $91.62 \pm 9.84$ \\
\hline & Week 4 & $67.48 \pm 20.17$ & $57.91 \pm 25.05$ & $171.50 \pm 29.85$ & $139.50 \pm 44.92$ \\
\hline & Week 12 & $46.43 \pm 13.73$ & $87.01 \pm 12.17$ & $28.25 \pm 2.37^{* * *}$ & $28.40 \pm 6.70^{* * *}$ \\
\hline
\end{tabular}

* - significance as compared to HFD alone group. $\left.{ }^{*}-<0.05 ;^{* \star *}-<0.001\right)$

$\wedge$ - significance as compared to normal control. $\left({ }^{\wedge \wedge \wedge}-<0.001\right)$ 
Table 3: Results of Ficus carica pulp on lipid parameters (mg/dl) in rats. HFD - high fat diet, FCP - Ficus carica pulp; ATO - atorvastatin.

\begin{tabular}{|l|l|l|l|l|l|}
\hline & & Normal Control & HFD alone & HFD+FCP & HFD + ATO \\
\hline \multirow{3}{*}{ HDL } & Week 0 & $25.80 \pm 2.84$ & $26.18 \pm 3.4$ & $25.87 \pm 2.64$ & $23.64 \pm 3.33$ \\
\cline { 2 - 6 } & Week 4 & $46.58 \pm 12.99$ & $48.94 \pm 9.97$ & $37.68 \pm 7.58$ & $31.55 \pm 4.51$ \\
\cline { 2 - 6 } & Week 12 & $29.39 \pm 7.94$ & $39.33 \pm 7.19$ & $52.19 \pm 2.17^{* *}$ & $43.79 \pm 9.67$ \\
\hline \multirow{3}{*}{ LDL } & Week 0 & $41.73 \pm 17.11$ & $48.62 \pm 12.98$ & $43.77 \pm 7.56$ & $42.25 \pm 5.38$ \\
\cline { 2 - 6 } & Week 4 & $24.76 \pm 13.61$ & $141.05 \pm 58.08$ & $179.69 \pm 32.3$ & $175.70 \pm 54.3$ \\
\cline { 2 - 6 } & Week 12 & $23.05 \pm 15.09$ & $168.56 \pm 44.18$ & $61.68 \pm 18.61^{* * *}$ & $92.83 \pm 17.76^{* * * \wedge \wedge}$ \\
\hline \multirow{3}{*}{ HDL/LDL } & Week 0 & $1.64 \pm 0.68$ & $1.92 \pm 0.71$ & $1.71 \pm 0.37$ & $1.82 \pm 0.36$ \\
\cline { 2 - 6 } & Week 4 & $0.66 \pm 0.56$ & $3.22 \pm 2.16$ & $4.95 \pm 1.33$ & $5.61 \pm 1.70$ \\
\cline { 2 - 6 } & Week 12 & $0.80 \pm 0.54$ & $4.55 \pm 1.97$ & $.18 \pm 0.38^{* * *}$ & $2.22 \pm 0.61^{* * *}$ \\
\hline \multirow{3}{*}{ AIP } & Week 0 & $.52 \pm 0.04$ & $.53 \pm 0.06$ & $.22 \pm 0.12$ & $.57 \pm 0.13$ \\
\cline { 2 - 6 } & Week 4 & $-.19 \pm 0.15$ & $.16 \pm 0.22$ & $-.26 \pm 0.04^{* * * \wedge \wedge}$ & $.08 \pm 0.32$ \\
\cline { 2 - 6 } & Week 12 & $.19 \pm 0.15$ & $.34 \pm 0.06$ & $-.18 \pm 0.12^{* * * \wedge \wedge}$ \\
\hline
\end{tabular}

* - significance as compared to HFD alone group. $\left({ }^{*}-<0.05 ;{ }^{* \star *}-<0.001\right)$

$\wedge$ - significance as compared to normal control. $\left({ }^{\wedge \wedge \wedge}-<0.001\right)$

HDL/LDL: Being highest in HFD alone group, this ratio significantly decreased in both HFD+FCP and HFD+ATO groups ( $p$ value $<0.001$ for both groups); the least was in HFD+FCP group with level being insignificantly different than normal control group. The difference among HFD+FCP and HFD+ATO groups themselves was though insignificant.

Atherogenic Index of Plasma (AIP): AIP was the highest in HFD alone group. Atorvastatin and Ficus carica pulp both decreased it significantly than HFD alone and even normal control groups ( $p$ value $<0.001$ ). Decrease of AIP in Ficus carica pulp group was maximum but statistically insignificant as compared to HFD+ATO group.

\section{DISCUSSION}

Hypercholesterolemia causes atherosclerosis and is the most important risk factor for cardiovascular disease. Cardiovascular disease is the leading cause of morbidity and mortality throughout the world which lead to casualities in both underdeveloped and developed countries. To reduce the mortality and morbidity serum cholesterol should be controlled ${ }^{15}$. Seeds and fruits of various plants have been studied for their antihyperlipidemic effect. Ficus carica has many medicinal uses. Leaves or fruit of ethanolic extracts of Ficus carica have been used to demonstrate hypolipidemic effect in previous studies ${ }^{16}$. As Ficus carica fruit is freely available and edible as such, its use is easier than extract whose extraction in itself is a lengthy process. This study, therefore, compaerd hypolipidemic effect of Ficus carica pulp and atorvastatin, a standard agent.

Male sprague-dawley rats were used because protective role of female sex hormones may interfere with hyperlipidaemia induction in female rats ${ }^{17}$. Induction of hyperlipidaemia was done by using cholesterol powder and coconut oil which have $90 \%$ saturated fats ${ }^{18}$.

A part from random variations in normal control lipid profile, also referenced in literature ${ }^{19}$, reduction in triglyceride level was significant and comparable in atorvastatin and Ficus carica pulp treated groups. Similar studies conducted on Ficus carica fruit extract ${ }^{16}$ and leaves have demonstrated reduced triglyceride level. Most of the studies showed that increased triglycerides is related with coronary artery disease, so reduction of triglycerides is very important. Reduction in serum total cholesterol in group treated with Ficus carica was also significant. The reduction in cholesterol is even lower as compared to the group treated with atorvastatin. Similar study using ethanolic extract of leaves of Ficus carica showed marked decrease in level of cholesterol ${ }^{20}$. In this study, when hyperlipidaemia was induced, it did not decrease HDL level significantly than normal control probably because of elevated total cholesterol. However, treatment with Ficus carica pulp significantly improved HDL levels than HFD alone while improvement in atorvastatin group was statistically insignificant. Increase in level of HDL was also shown by similar study which was done on leaf extract of Ficus carica $^{21}$. HDL is important because of its established cardioprotective role of HDL in various studies. Cholesterol from the arteries is transported to the liver by HDL and oxidation of LDL in liver is prevented. In this study, HDL in Ficus carica treated group was greater than HFD group and also higher than normal control rats. Other studies conducted on fenugreek seed extract and rimonabant also exhibited increase in $\mathrm{HDL}^{22}$. Level of LDL in Ficus carica treated group was decreased and it is comparable to atorvastatin. Similar studies which were done on antihyperlipidemic effects of Ficus carica fruit and Ficus carica wine from its fruit and leaves showed decrease in LDL level supporting the results of present study ${ }^{23}$. LDL/HDL ratio in Ficus carica pulp and atorvastatin treated groups showed comparable and significant reduction. Similar studies done on Ficus carica on experimental animals also showed reduced LDL/HDL ratio ${ }^{21}$. Decrease of atherogenic index of plasma with Ficus carica pulp was equivalent to atorvastatin. One study which was done on leaves and fruit of Ficus carica showed reduction in AIP9. Body weight steadily increased in all groups in present study. It was maximum in atorvastatin treated group at end of the study while this difference was statistically insignificant than Ficus carica pulp treated group. Similar studies on leaf and fruit of Ficus carica extracts showed a persistent increase in weight of rats ${ }^{24}$.

Fruit of Ficus carica contains many compounds like flavonoids, phytosterols, phenols and anthracyanin pigments. Ficus carica leaves also contain many compounds like organic acids, phenolic acids, oleanic acid and polyphenols. Antioxidant activity of Ficus carica may be due to the prescence of these compounds ${ }^{25}$.

Hence it is assumed that Ficus carica pulp has antihyperlipidemic effect. This effect is most probably due to high anthracyanin pigments, flavonoids and polyphenols 
and these componds have radical scavenging and antioxidant activity. More research should be required to find the mechanism of antihyperlipidemic effect of Ficus carica.

\section{CONCLUSION}

Hypolipidemic effect of pulp of Ficus carica is comparable to atorvastatin. It may be alternative to statins due to lack of side effects.

Acknowledgement: Authors are thankful to the staff of Animal house and Chemical Pathology Laboratory of PGMI, Lahore for their cooperation.

Conflict of Interest: The authors declare no conflict of interest.

Source of Funding: Post Graduate Medical Institute, Lahore

Disclaimer: Data is part of M. Phil thesis.

\section{REFERENCES}

1. Raza Q, Doak CM, Khan A, Nicolaou M, Seidell JC. Obesity and cardiovascular disease risk factors among the indigenous and immigrant Pakistani population: a systematic review. Obes Facts. 2013;6(6):523-35.

2. Basharat Z, Mumtaz S, Rashid F, Rashid S, Mallam SA, Diljan A, Iftikhar-Maken N, Zafar S, Rehman I. Prevalence of risk factors of ischemic stroke in a local Pakistani population. High-density lipoproteins, an emerging risk factor. Neurosciences (Riyadh). 2012 Oct;17(4):357-62.

3. Gupta A, Sehgal V, Mehan S. Hyperlipidaemia: An updated review. International journal of Biopharmaceutical Toxicol Res. 2011;(1):81-89.

4. Van Himbergen TM, Otokozawa S, Matthan NR, Schaefer EJ, Buchsbaum A, Ai M, van Tits LJ, de Graaf J, Stalenhoef AF. Familial combined hyperlipidemia is associated with alterations in the cholesterol synthesis pathway. Arterioscler Thromb Vasc Biol. 2010 Jan;30(1):113-20.

5. Mahmood Z, Ahmed W, Sualeh M, Mehmood S. Hperlipidemia development \& consequences. Med Chhanel. 2009; 15(3):14-17.

6. Steinberg D, Glass CK, Witztum JL. Evidence mandating earlier and more aggressive treatment of hypercholesterolemia. Circulation. 2008 Aug 5;118(6):672-7.

7. Mampuya WM, Frid D, Rocco M, Huang J, Brennan DM, Hazen SL, Cho L. Treatment strategies in patients with statin intolerance: the Cleveland Clinic experience. Am Heart J. 2013 Sep;166(3):597-603.

8. Razavi S M, Pourfarzad A, Sourky A H, Jahromy S. The physical properties of fig (ficus carica L.) as a function of moisture content and variety. PHILIP AGRIC SCIENTIST. 2010;93(2):170-181.

9. Jeong M, Han J, Hwang E, Lee Y. Fig wine, fig fruits and fig leaves improve lipid profile and suppress body weight inrats high-fat diet. FASEB J. 2007;21(6):846.6.

10. Rassouli A, Fatemi Ardestani A, Asad F. Effects of fig tree(Ficus Carica) leaf extract on serum and liver cholesterol levels in hyperlipidemic rats. Int J Vet Res. 2010;4(2): 77-80.
11. Bayne K. Revised Guide for the care and use of laboratory animals. American Physiological Society. The Physiologist. 1996;39(4):199-208.

12. Balasubramanian $M N$, Muralidharan $P$, Balamurugan $G$ Anti hyperlipidemic activity of Pedalium murex (Linn.) Fruits on High Fat Diet Fed Rats. International journal of pharmacology. 2008;4(4):310-313.

13. Kannur D, Khandelwal K. Phytochemical investigation and evaluation of antihyperlipidemic activity of Ficus Carica Linn fruit extracts. GJRA. 2014;3(6):2277-8160.

14. Ji G, Zhao X, Leng L, Liu P, Jiang Z. Comparison of dietary control and atorvastatin on high fat diet induced hepatic steatosis and hyperlipidemia in rats. Lipids Health Dis. 2011 Jan 26;10:23.

15. Tietge UJ. Hyperlipidemia and cardiovascular disease: inflammation, dyslipidemia, and atherosclerosis. Curr Opin Lipidol. 2014 Feb;25(1):94-5.

16. Asad F, Pourkabir M, Maclaren R, Shahriari A. Alteration to lipid parameters in response to fig tree(Ficus carica) leaf extract in chicken liver slices. Turk $\mathrm{J}$ Vet Anim Sci. 2006;30:315-318.

17. Getz G, Reardon C. Animal models of atherosclerosis. ATVB. 2012;2(5):1104-1115.

18. Munshi RP, Joshi SG, Rane BN. Development of an experimental diet model in rats to study hyperlipidemia and insulin resistance, markers for coronary heart disease. Indian J Pharmacol. 2014 May-Jun;46(3):270-6.

19. Ihedioha J I, Noel-Uneke O A, Ihedioha, T E. Reference values for the serum lipid profile of albino rats (rattus norvegicus) of varied ages and sexes. Comp Clin Pathol. 2013;22:93-99.

20. Allahyari S, Delazar A, Najafi M. Evaluation of general toxicity, anti-oxidant activity and effects of ficus carica leaves extract on ischemia/reperfusion injuries in isolated heart of rat. Adv Pharm Bull. 2014 Dec;4(Suppl 2):577-82.

21. Joerin L, Kauschka M, Bonnländer B, Pischel I, Benedek B, Butterweck V. Ficus carica leaf extract modulates the lipid profile of rats fed with a high-fat diet through an increase of HDL-C. Phytother Res. 2014 Feb;28(2):261-7.

22. Sharma MS, Choudhary PR. Hypolipidemic effect of fenugreek seeds and its comparison with atorvastatin on experimentally induced hyperlipidemia. J Coll Physicians Surg Pak. 2014 Aug;24(8):539-42.

23. Belguith-Hadriche O, Ammar S, Contreras Mdel M, Turki M, Segura-Carretero A, El Feki A, Makni-Ayedi F, Bouaziz M. Antihyperlipidemic and Antioxidant Activities of Edible Tunisian Ficus carica L. Fruits in High Fat Diet-Induced Hyperlipidemic Rats. Plant Foods Hum Nutr. 2016 Jun;71(2):183-9.

24. Jeong M, Kim H, Cha J D. Antibacterial activity of Methanol extract from Ficus Carica leaves against oral bacteria. $\mathrm{J}$ of Bact \& Virol. 2009;39(2):97-102.

25. Ghazi $F$ et al., Determination of total polyphenols \& nutritional composition of two different types of Ficus carica leaves cultivated in saudi arabia. PAK $J$ Nutr. 2012;11(11):1061-1065. 